\title{
Study of the sharp "knee" phenomenon of cosmic ray spectrum by using newly upgraded Tibet $\mathbf{A S} \gamma$ experiment
}

\author{
Ying Zhang ${ }^{* 1}$, J. Huang ${ }^{1}$, D.Chen ${ }^{2}$, L. M. Zhai ${ }^{2}$, Xu Chen $^{1}$, Yu-Hui Lin ${ }^{1}$, Jian-Hua \\ Fang $^{1}$, Yoshiaki Nakamura ${ }^{1}$ \\ ${ }^{1}$ Key Laboratory of Particle Astrophysics, Institute of High Energy Physics, Chinese Academy of \\ Sciences, Beijing 100049, China \\ ${ }^{2}$ National Astronomical Observatories, Chinese Academy of Sciences, Beijing 100012, China \\ E-mail: vingzhangdihep.ac.cn
}

\begin{abstract}
Cosmic Ray (CR) spectrum in a certain energy region can not be expressed by a simple power-law as it has a structure. The all-particle energy spectrum of primary CRs observed in a wide range from $10^{14} \mathrm{eV}$ to $10^{17} \mathrm{eV}$ with the Tibet-III AS array clearly shows a 'sharp knee' at around 4 $\mathrm{PeV}$. Based on the hardening structure of energy spectrum observed by AMS-02, CREAM and the (proton+helium) spectrum at $50-1000 \mathrm{TeV}$ energy region observed by (YAC-I+Tibet-III), we will discuss the origin of 'shap knee' whether it is caused by nearby source or nonlinear acceleration.
\end{abstract}

36th International Cosmic Ray Conference -ICRC2019-

July 24th - August 1st, 2019

Madison, WI, U.S.A.

\footnotetext{
* Speaker.
} 


\section{Acknowledgements}

The authors would like to express their thanks to the members of the Tibet AS $\gamma$ collaboration for fruitful discussions. This work is supported by the National Key R\&D Program of China (No.2016YFE0125500), by the National Natural Science Foundation of China (Nos. 11803038, 11533007, 11673041, 11603047 and 11873065) and by the Key Laboratory of Particle Astrophysics, Institute of High Energy Physics, CAS. 